\title{
ON GLOBAL REPRESENTATIONS OF THE SOLUTIONS OF LINEAR DIFFERENTIAL EQUATIONS AS A PRODUCT OF EXPONENTIALS
}

\author{
J. WEI AND E. NORMAN
}

1. Introduction. In this paper we shall consider solutions of the equation

$$
\frac{d U(t)}{d t}=A(t) U(t), \quad U(0)=I,
$$

where $A$ and $U$ are linear operators, and $I$ is the identity operator. Our results will be applicable when the operator $A(t)$ can be written as

$$
A(t)=\sum_{i=1}^{m} a_{i}(t) X_{i}, \quad m \text { finite }
$$

where the $a_{i}(t)$ are scalar functions of $t$, and the operators $X_{i}$ are independent of $t$. It is further required that the Lie algebra $\mathscr{L}$ generated by the $X_{i}$ under the commutator product $\left[X_{i}, X_{j}\right]=X_{i} X_{j}$ $-X_{j} X_{i}$ be of finite dimension $l$. The above is, of course, always true if $A$ (and $U$ ) are finite matrix operators.

In 1954, W. Magnus [4] proved that if $X_{1}, X_{2}, \cdots, X_{l}$ is a basis for $\mathscr{L}$, then the solution of (1) can be expressed in the form $U(t)$ $=\exp \left(\sum_{i=1}^{l} g_{i}(t) X_{i}\right)$. This representation of $U$ holds, however, only in a neighborhood of the origin. It has been shown by J. Mariani and W. Magnus [3] that even in the case of $2 \times 2$ matrices a global version of Magnus' result cannot be obtained without severe restrictions on $A(t)$.

We will show that if $U$ is a solution of (1), it can be represented in the form

$$
U(t)=\prod_{i=1}^{l} \exp \left(g_{i}(t) X_{i}\right)
$$

This representation is global for all solvable Lie algebras, and for any real $2 \times 2$ system of equations.

The form (2) derives its principal utility from the fact that insight into the properties of $U(t)$ can be gained through a knowledge of the spectral properties of the individual operators $X_{i}$. Since the $X_{i}$ 's are

Received by the editors July 26, 1962 and, in revised form, January 17, 1963. 
constant, and often have simple physical interpretations, one has a good chance of obtaining their spectral properties.

2. Preliminaries. In the sequel it will be convenient to refer to the independent variable as "time."

Suppose the linear operator $A(t)$ can be expressed in the form

$$
A(t)=\sum_{i=1}^{m} a_{i}(t) X_{i}, \quad m \text { finite }
$$

where the $a_{i}(t)$ are scalar functions of time, and $X_{1}, X_{2}, \cdots, X_{m}$ are time-independent operators. If $m$ is chosen as small as possible, the $X_{i}$ 's will be linearly independent. We shall denote by $R$ the associative algebra generated by $X_{1}, X_{2}, \cdots, X_{m}$ over the field of complex numbers; and by $\mathscr{L}$ the Lie algebra generated by $X_{1}, \cdots, X_{m}$ under the commutator product $\left[X_{i}, X_{j}\right]=X_{i} X_{j}-X_{j} X_{i}$, i.e., the Lie elements of $R$. $R$ may be infinite-dimensional, but we shall assume that $\mathfrak{L}$ is of finite dimension $l$.

We will require the following two lemmas.

(i) (BAKer-HAusdorfF). If $, x, y \in \mathcal{L}$, then $e^{x} y e^{-x} \in \mathcal{L}$, and is given by the explicit formula

(4) $e^{x} y e^{-x}=y+[x, y]+[x,[x, y]] / 2 !+[x[x,[x, y]]] / 3 !+\cdots$.

A proof is given in Magnus [4].

If we use the notation, $a d x$, to represent the linear operator on $\mathfrak{L}$ defined by

$$
\begin{aligned}
(a d x) y & =[x, y] \\
(a d x)^{2} y & =[x,[x, y]], \text { etc. } \quad x, y \in \mathcal{L},
\end{aligned}
$$

then the Baker-Hausdorff formula (4) can be rewritten as

$$
e^{x} y e^{-x}=\left(e^{a d x}\right) y .
$$

(ii) Let $X_{1}, X_{2}, \cdots, X_{l}$ be a basis for $\mathfrak{L}$ with the multiplication table $\left[X_{i}, X_{j}\right]=\sum_{k=1}^{l} \gamma_{i j}^{k} X_{k}, i, j=1,2, \cdots, l$. Then

$$
\begin{aligned}
\left(\prod_{j=1}^{r} \exp \left(g_{j} X_{j}\right)\right) X_{i}\left(\prod_{j=r}^{1} \exp \left(-g_{j} X_{j}\right)\right)=\sum_{k=1}^{l} \xi_{k i} X_{k}, & \\
r & =1,2, \cdots, l,
\end{aligned}
$$

where each $\xi_{k i}=\xi_{k i}\left(g_{1}, \cdots, g_{r}\right)$ is an analytic function of $g_{1}$ to $g_{r}$.

Proof. Repeated application of the previous lemma shows that the left-hand side of $(6)$ is in $\mathscr{L}$, and hence can be written as a linear 
combination of $X_{1}$ to $X_{l}$ as asserted. It remains to show that the $\xi_{k i}$ are analytic. It is sufficient to prove the result for $r=1$ since an analytic function of an analytic function is again analytic. For $r=1$ we have from (4)

$$
\begin{aligned}
\exp \left(g_{1} X_{1}\right) X_{i} \exp \left(-g_{1} X_{1}\right) & =\exp \left(g_{1} a d X_{1}\right) X_{i} \\
& =X_{i}+\sum_{n=1}^{\infty} \frac{g_{1}^{n}}{n !}\left(\operatorname{ad} X_{1}\right)^{n} X_{i} .
\end{aligned}
$$

Now

(7) $\left(a d X_{1}\right)^{n} X_{i}=\sum \gamma_{1_{i}}^{i_{1}} \gamma_{1_{i_{1}}}^{i_{2}}, \cdots, \gamma_{1_{i_{n+1}}}^{i_{n}} X_{i_{n}}, \quad i_{1}, \cdots, i_{n}=1, \cdots, l$.

Let $M$ be the maximum of $\left|\gamma_{i j}^{k}\right|, i, j, k=1, \cdots, l$. Estimating each $\gamma_{i_{i_{s-1}}}^{i_{s}}$ in (7) by $M$ we obtain

$$
\left|\sum \gamma_{i_{i_{1}}}^{i_{1}} \cdots\right| \leqq(l M)^{n}
$$

Since $(l M)^{n} / n$ ! is the general term of a convergent series the lemma is proved.

\section{The local theorem.}

ThEOREM 1. Let $A(t)$ be given by (3), and let the Lie algebra $\&$ generated by $A(t)$ be of finite dimension $l$. Then there exists a neighborhood of $t=0$ in which the solution of the equation

$$
\frac{d U}{d t}=A(t) U, \quad U(0)=I
$$

may be expressed in the form

$$
U(t)=\exp \left(g_{1}(t) X_{1}\right) \exp \left(g_{2}(t) X_{2}\right) \cdots \exp \left(g_{l}(t) X_{l}\right),
$$

where the $g_{i}(t)$ are scalar functions of time. Moreover, the $g_{i}(t)$ satisfy a set of differential equations which depend only on the Lie algebra $\mathfrak{L}$, and the $a_{i}(t)$ 's.

Proof. The representation (9) is immediate from the Magnus representation via the second canonical coordinate system. We will, however, prove this fact again in the course of deriving the differential equations satisfied by the $g_{i}(t)$.

First note that we might just as well write $A(t)=\sum_{i=1}^{l} a_{i}(t) X_{i}$ instead of $A(t)=\sum_{i=1}^{m} a_{i}(t) X_{i}$ by simply setting $a_{i}(t) \equiv 0$ for $i>m$. Note also that at time $t=0, U(0)=I$ is in the form (9) with all $g_{i}(t)$ $=0$. 
Now let $U$ be of the form (9). Since

$$
\begin{aligned}
& \frac{d U}{d t}=\sum_{i=1}^{l} g_{i}^{\prime}(t)\left(\prod_{j=1}^{i-1} \exp \left(g_{j} X_{j}\right) X_{i} \prod_{j=i}^{l} \exp \left(g_{j} X_{j}\right)\right) \\
& A U=\sum_{i=1}^{l} a_{i}(t) X_{i} \cdot U .
\end{aligned}
$$

We obtain upon substitution of (10) into (8), and post-multiplication by the inverse operator $U^{-1}$

$$
\begin{aligned}
\sum_{i=1}^{l} a_{i}(t) X_{i} & =\sum_{i=1}^{l} g_{i}^{\prime}(t)\left(\prod_{j=1}^{i-1} \exp \left(g_{j} X_{j}\right) X_{i} \prod_{j=i-1}^{1} \exp \left(-g_{j} X_{j}\right)\right) \\
& =\sum_{i=1}^{l} g_{i}^{\prime}(t)\left(\prod_{j=1}^{i-1} \exp \left(g_{j} a d X_{j}\right)\right) X_{i} .
\end{aligned}
$$

Application of Lemma (ii) to the terms on the right of equation (11) yields

$$
\sum_{k=1}^{l} a_{k}(t) X_{k}=\sum_{i=1}^{l} \sum_{k=1}^{l} g_{i}^{\prime}(t) \xi_{k i} X_{k} .
$$

Since the operators $X_{k}$ are linearly independent we have a linear relation between the $a_{k}(t)$ and the $g_{i}^{\prime}(t)$. The elements $\xi_{k i}$ of the transform matrix $\xi$ are analytic functions of the $g_{i}$ 's.

$$
\left[\begin{array}{c}
a \\
a_{1} \\
a_{2} \\
\vdots \\
a_{l}
\end{array}\right]=\left[\begin{array}{ccc}
\xi & & g_{11} \\
& \cdots & \xi_{1 l} \\
& & \\
& & \\
\xi_{l 1} & & \xi_{l l}
\end{array}\right]\left[\begin{array}{c}
g_{1}^{\prime} \\
g_{2}^{\prime} \\
\vdots \\
g_{l}^{\prime}
\end{array}\right], \quad g(0)=0 .
$$

Since the $\xi_{k i}$ are analytic functions of $g$, we have that the determinant $\Delta$ of $\xi$ is an analytic function of $g$. We also know that at $t=0, \xi=I$, and hence $\Delta(0) \neq 0$. These two facts show that there must exist a neighborhood $N_{0}$ of $t=0$ in which $\Delta \neq 0$, i.e., in which $\xi$ is invertible. We can thus write (13) in the form

$$
\frac{d g}{d t}=f(a, g)=\xi^{-1} a, \quad g(0)=0, \quad t \in N_{0} .
$$

Since $\xi^{-1}$ is analytic in $N_{0}$, we are assured of a neighborhood of $t=0$ in which the solution of (14) exists and is unique. This completes the proof of the theorem. 
EXAMPLE. Let $d U / d t=\left(a_{1}(t) H+a_{2}(t) E+a_{3}(t) F\right) U$, where $H, E$, and $F$ are constant linear operators with the Lie multiplication table $[E, F]=H ;[E, H]=2 E ;[F, H]=-2 F$. Setting $U=e^{\sigma_{1}(t) H} e^{\sigma_{2}(t) E} e^{\sigma_{3}(t) F}$, we obtain

$$
\begin{aligned}
a_{1} H+a_{2} E+a_{3} F & =g_{1}^{\prime} H+g_{2}^{\prime}\left(e^{\sigma_{1} a d H}\right) E+g_{3}^{\prime}\left(e^{\sigma_{1} a d H} e^{g_{2} a d E}\right) F \\
\left(e^{\sigma_{1} a d H}\right) E & =E-2 g_{1} E+\frac{\left(2 g_{1}\right)^{2}}{2 !} E-\cdots=e^{-2 \sigma_{1}} E \\
\left(e^{\sigma_{1} a d H} e^{\sigma_{2} a d E}\right) F & =\left(e^{\sigma_{1} a d H}\right)\left(F+g_{2} H+g_{2}^{2} E\right) \\
& =e^{2 \sigma_{1} F}+g_{2} H+g_{2}^{2} e^{-2 \sigma_{1}} E .
\end{aligned}
$$

Hence $g_{1}, g_{2}, g_{3}$ satisfy the equations

$$
\left[\begin{array}{l}
a \\
b \\
c
\end{array}\right]=\left[\begin{array}{lll}
1 & 0 & g_{2} \\
0 & e^{-2 g_{1}} & g_{2}^{2} e^{-2 g_{1}} \\
0 & 0 & e^{2 g_{1}}
\end{array}\right]\left[\begin{array}{l}
g_{1}^{\prime} \\
g_{2}^{\prime} \\
g_{3}^{\prime}
\end{array}\right] .
$$

\section{Global results.}

THEOREM 2. If $\&$ is solvable, then there exists a basis, and an ordering of this basis, for which Theorem 1 is global.

Proof. A theorem of Lie states that if $\mathscr{L}$ is solvable, then there exists a chain of ideals $0 \subset L_{l} \subset L_{l-1} \subset \cdots \subset L_{1}=\mathscr{L}$, where each $L_{m}$ is exactly of dimension $l-m+1$. It is easy to see that there is a basis $X_{1}, X_{2}, \cdots, X_{l}$ for $\&$ which can be arranged so that $L_{m}$ is the ideal generated by $X_{m}, \cdots, X_{l}$. With this arrangement the multiplication table for $\mathscr{L}$ becomes

$$
\left[X_{i}, X_{j}\right]=\sum_{k=i}^{l} \gamma_{i j}^{k} X_{k} \quad \text { for } i>j .
$$

Since $\left(\prod_{j=1}^{i-1} \exp \left(g_{j} a d X_{j}\right)\right) X_{i}=\sum_{k=1}^{l} \xi_{k i} X_{k}=\sum_{k=i}^{l} \xi_{k i} X_{k}$, we have that $\xi_{k i} \equiv 0$ for $i>k$. It is clear that $\xi_{k i}$ depends only on $g_{j}$ with $j<k$; thus system $\xi g^{\prime}=a$ is in triangular form. To show that $\xi g^{\prime}=a$ has a solution for all $t$ for which $a(t)$ is continuous, it is sufficient to prove that the diagonal elements $\xi_{i i}$ never vanish. We shall show that $\xi_{i i}$ $=\exp \left(\sum_{j=1}^{i-1} g_{j} \gamma_{j i}^{i}\right)$. This is readily seen since, by use of Lemma (i), one finds that

$$
\begin{aligned}
\exp \left(g_{i-1} a d X_{i-1}\right) X_{i}= & X_{i}+g_{i-1} \gamma_{i-1, i}^{i} X_{i}+\text { terms in } L_{i+1} \\
& +\left(g_{i-1} \gamma_{i-1, i}^{i}\right)^{2} / 2 ! X_{i}+\text { terms in } L_{i+1}+\cdots \\
= & X_{i} \exp \left(g_{i-1} \gamma_{i-1, i}^{i}\right)+\text { terms in } L_{i+1} .
\end{aligned}
$$


The proof is completed by repetition of the above argument.

There is an interesting formula due to Zassenhaus [see 4, p. 661] which states that if $X, Y$ generate the free algebra $\mathcal{L}$, then $e^{X+Y}$

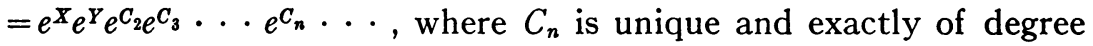
$n$ in $X, Y$. Magnus gives a method by which the $C_{n}$ may be found recursively. For solvable algebras Theorem 2 yields the following sharpening of the Zassenhaus formula.

Corollary. If $X_{1}, \cdots, X_{m}$ generate a solvable Lie algebra $\mathfrak{L}$ of dimension $l$, then

$$
\exp \left(\sum_{i=1}^{m} a_{i} X_{i}\right)=\prod_{i=1}^{l} \exp \left(g_{i} Y_{i}\right),
$$

where $Y_{1}, \cdots, Y_{l}$ is a suitable basis for $\mathcal{L}$. The $g_{i}$ can be found by quadrature.

Theorem 2 allows us to confine our attention to the case where $\mathscr{L}$ is semisimple. To see this we make use of Levi's theorem (see Jacobson $\left[2\right.$, p. 91]); $\mathfrak{L}$ may be decomposed into the direct sum $\mathscr{L}=L_{0}+L_{1}$, where $L_{0}$ is the radical of $\mathscr{L}$, and $L_{1}$ is a semisimple subalgebra of $\mathscr{L}$. In the equation $d U / d t=A(t) U$, where $A(t)$ generates $\mathcal{L}$, the decomposition $\mathscr{L}=L_{0}+L_{1}$ gives rise to the corresponding decomposition, $A=A_{0}+A_{1}$ of $A$, where $A_{i}$ is in $L_{i}$. If we have proved that Theorem 1 is global for all semisimple Lie algebras as well as all solvable Lie algebras, then the following scheme extends the result to $\mathscr{L}$. Let

$$
\frac{d U}{d t}=A U=\left(A_{0}+A_{1}\right) U
$$

Define $U_{0}$, and $U_{1}$, by $d U_{1} / d t=A_{1} U_{1}, d U_{0} / d t=\left(U_{1}^{-1} A_{0} U_{1}\right) U_{0}$. Since $L_{0}$ is an ideal in $\mathscr{L}$, we see (by Lemma (i)) that $U_{1}^{-1} A_{0} U_{1}$ is in $L_{0}$. It is easy to verify that $U=U_{1} U_{0}$ satisfies (16).

If $\mathcal{L}$ is semisimple, the global nature of Theorem 1 is in doubt. The examples below show that the choice of basis is critical.

Example 1. Let $d U / d t=A U$ with $A$ a $3 \times 3$ antisymmetric matrix. $A$ generates the Lie algebra $X_{1}=E_{12}-E_{21}, X_{2}=E_{13}-E_{31}, X_{3}=E_{23}$ $-E_{32},\left[X_{1}, X_{2}\right]=-X_{3},\left[X_{1}, X_{3}\right]=X_{2},\left[X_{2}, X_{3}\right]=-X_{1}$. If we assume a solution of the form $U=e^{\theta_{1} X_{1}} e^{\rho_{2} X_{2}} e^{\theta_{3} X_{3}}$ and compute the corresponding $\xi$ matrix, we find that $|\xi|=\cos g_{2}$. Hence $\xi$ is not invertible for all time, and the representation is not global. We get the same result for any ordering of the above basis.

EXAMPLE 2. This example shows that even if $\xi$ is invertible for all time, the representation by a product of exponentials need not be 
global. Let $d U / d t=A U$ with $A$ continuous for $0 \leqq t<\infty, a_{11}=-a_{22}$, and

$$
A=\left[\begin{array}{rr}
-6 & -4 \\
5 & 6
\end{array}\right] \quad \text { for } 0 \leqq t<1 \text {. }
$$

The Lie algebra generated by $A$ is the same as that given in the example of $\S 3$. If we assume a solution of the form $U=\exp \left(g_{1} H\right) \exp \left(g_{2} E\right)$ $\exp \left(g_{3} F\right)$, we find that for $0 \leqq t<1, e^{g_{1}}=\cosh 4 t-(3 / 2) \sinh 4 t$. Thus, $g_{1}(t)=-\infty$ at $t=(1 / 8) \ln 5$, and the representation is not global. In this example $|\xi| \equiv 1$ so that $\xi$ is always invertible.

The next example is interesting enough to state as a theorem.

TheOREM 3. If $d U / d t=B U$, where $B$ is any real continuous $2 \times 2$ matrix, then $U$ has the form $U=\exp \left(g_{1} K\right) \exp \left(g_{2} A\right) \exp \left(g_{3} N\right) \exp \left(g_{4} I\right)$, where $K=E_{12}-E_{21}, A=E_{11}-E_{22}, N=E_{12}$, and $I$ is the identity; this representation is global.

Proof. Let $B=S+a I, S$ generates the algebra $\{K, A, N\}$. Then $U$ has the form $V e^{\sigma_{4} I}$ with $g_{4}=\int_{0}^{t} a(\tau) d \tau$ and $d V / d t=S V$. Theorem 1 shows that $V$ has the required form in a neighborhood of the origin. Now,

$$
\begin{aligned}
\exp \left(g_{1} K\right) & \exp \left(g_{2} A\right) \exp \left(g_{3} N\right) \\
= & {\left[\begin{array}{rr}
e^{\sigma_{2}} \cos g_{1} & g_{3} e^{\theta_{2}} \cos g_{1}+e^{-\sigma_{2}} \sin g_{1} \\
-e^{\sigma_{2}} \sin g_{1} & -g_{3} e^{\sigma_{2}} \sin g_{1}+e^{-\sigma_{2}} \cos g_{1}
\end{array}\right]=\left[\begin{array}{ll}
V_{11} & V_{12} \\
V_{21} & V_{22}
\end{array}\right] . }
\end{aligned}
$$

Solving for the $g_{i}$ in terms of the $V_{i j}$ gives $g_{1}=-\operatorname{Arctan}\left(V_{21} / V_{11}\right)$, $g_{2}=(1 / 2) \ln \left(V_{11}^{2}+V_{21}^{1}\right), g_{3}=\left(V_{11} V_{12}+V_{21} V_{22}\right) /\left(V_{11}^{2}+V_{21}^{2}\right)$. Since $V$ is nonsingular for all time, $V_{11}$ and $V_{21}$ cannot vanish simultaneously. Hence the $g_{i}$ are analytic functions of the $V_{i j}\left(V_{i j}\right.$ assumed real). This completes the proof.

If $d U / d t=A U$, where $A$ generates $\mathscr{L}=\left\{X_{1}, \cdots, X_{l}\right\}$, then we may consider the representation $U=\prod \exp \left(g_{i} X_{i}\right)$ as a transformation of coordinates. In order that the above representation be global, it is clearly necessary and sufficient that (a) the mapping of the Lie algebra $\mathscr{L}$ to the solution space of $d U / d t=A U$ is onto, and (b) the Jacobian, $J(U / g)$, of the transformation has rank $l$ for all time. The second condition is fairly easy to investigate.

Theorem 4. Let $d U / d t=A U=\left(\sum_{i=1}^{l} a_{i} X_{i}\right) U$, and let $\xi$ be the matrix of Theorem 1. Then the rank of $J$ is the same as the rank of $\xi$ whenever the two matrices are defined (i.e., whenever $g_{1}, \cdots, g_{l}$ are bounded). 
ProOF.

$$
\begin{aligned}
\frac{\partial U}{\partial g_{k}} & =\prod_{i=1}^{k-1} \exp \left(g_{i} X_{i}\right) X_{k} \prod_{i=k}^{l} \exp \left(g_{i} X_{i}\right) \\
\frac{\partial U}{\partial g_{k}} U^{-1} & =\left(\prod_{i=1}^{k-1} \exp \left(g_{i} a d X_{i}\right)\right) X_{k}=\sum_{i=1}^{l} \xi_{i k} X_{i} .
\end{aligned}
$$

Therefore, $J U^{-1}=\xi X$, where $X$ is the "vector" $\left(X_{1}, \cdots, X_{l}\right)^{T}$. Since $U^{-1}$ is nonsingular, $J$ and $\xi$ must have the same rank.

The next theorem shows that there do exist bases for which $\xi$ is invertible.

TheOREM 5. Let $\&$ be a split Lie algebra of dimension l. Then $\mathfrak{L}$ can be decomposed, (e.g. the root space decomposition), into $\mathfrak{L}=L_{1} \oplus L_{2}$ where $L_{1}$ and $L_{2}$ are solvable subalgebras. There exists a basis, and an ordering of this basis, for which the matrix $\xi$ of Theorem 1 is always nonsingular.

Proof. For $L_{1}$ choose the basis and ordering as in Theorem 2, say $X_{1}, \cdots, X_{m}$. Then $\xi$ will have the form

$$
\xi=\left[\begin{array}{ll}
A & * \\
0 & B
\end{array}\right],
$$

where $A$ is upper triangular and nonsingular. Hence $\xi$ is invertible if $B$ is. Let $Y_{1}, \cdots, Y_{n}$ be a basis for $B$. The $k$ th column of $B$ consists of the coefficients of $Y_{1}, \cdots, Y_{n}$ in the expansion of $\left(\prod_{i=1}^{m} \exp \left(a d g_{i} X_{i}\right)\right)\left(\prod_{j=1}^{k-1} \exp \left(a d h_{j} Y_{j}\right)\right) Y_{k}$. Now, the operator $\prod_{i=1}^{m} \exp \left(a d g_{i} X_{i}\right)$ is an automorphism of $\mathscr{L}$, so that it is enough to show that the matrix $B_{1}$ with $k$ th column the coefficients of $Y_{1}, \cdots, Y_{n}$ in the expansion of $\left(\prod_{j=1}^{k-1} \exp \left(a d h_{j} X_{j}\right)\right) Y_{k}$ is nonsingular. $B_{1}$ is just the $\xi$-matrix for the algebra $L_{2}$, and since $L_{2}$ is solvable, $Y_{1}, \cdots, Y_{n}$ can be chosen so that $B_{1}$ is nonsingular.

\section{REFERENCES}

1. N. Bourbaki, Éléments de mathématique. Algèbres de Lie, Actualités Sci. Ind. No. 26, Hermann, Paris, 1961.

2. N. Jacobson, Lie algebras, Interscience, New York, 1962.

3. J. Mariani and W. Magnus, The exponential solution for the homogeneous linear differential equation of the second order, Research report no. BE-37, New York University, 1961.

4. W. Magnus, On the exponential solution of differential equations for a linear operator, Comm. Pure Appl. Math. 7 (1954), 649-673.

Socony Mobil Oil Company, Inc. 